\title{
Enhancement in inverse pyramid SERS substrates with entrapped gold nanoparticles
}

\author{
I. Rigó ${ }^{1}$, M. Veres ${ }^{1}$, Zs. Pápa ${ }^{1}$, L. Himics ${ }^{1}$, O. Hakkel ${ }^{2}$, P. Fürjes ${ }^{2}$ \\ 'Institute for Solid State Physics and Optics, Wigner Research Centre for Physics, Budapest, \\ Hungary \\ IInstitute of Technical Physics and Materials Science, Centre for Energy Research, Budapest, \\ Hungary
}

\begin{abstract}
Giant plasmonic surface enhancement has been observed in gold coated micron sized inverse pyramids entrapping a gold nanoparticle. The amplification of both surface enhanced Raman and photoluminescence signals was found to be dependent on the diameter of trapped gold nanoparticle and around 50-fold enhancement was detected for $250 \mathrm{~nm}$ diameter sample relatively to the $50 \mathrm{~nm}$ one. Finite differential time domain simulations, performed to determine the near-field distribution in the structure, showed that when the nanoparticle protrudes into the hotspot zone of the void, coupling of electromagnetic field occurs and the plasmon-related nearfield enhancement is concentrated into the close vicinity of the nanoparticle, mainly into the close gaps around the tangential points of the curved sphere and the flat pyramid surface. This results in a more than 15 times increase of the near-field intensity, compared to the empty void.
\end{abstract}

Keywords: Surface-enhanced Raman spectroscopy (SERS); surface plasmons; FiniteDifference Time-Domain (FDTD) method; electromagnetic (EM) enhancement

\section{Introduction}

Raman scattering is an inelastic light scattering process involving interaction of the incident photons with fundamental vibrations of the molecule (or phonons in case of crystals). The measured energy loss of the scattered photon provides information on the bonding configuration and so on the composition of the analyte. While the cross-section of Raman scattering is fairly weak (it can be $10^{12}-10^{14}$ times smaller than that of fluorescence [1]), it can remarkably be improved by implementing surface-enhanced Raman scattering (SERS) [2], where the degree of achievable sensitivity can reach attomolar $\left(10^{-18} \mathrm{M}\right)$ concentrations [3]. The single-molecule sensitivity of SERS has been demonstrated by many authors [4, 5].

SERS is based on the enhancement of the electromagnetic field of incident and/or scattered light during the interaction with surface plasmons in close vicinity of metallic surfaces [6]. Efficient enhancement requires the surface plasmon resonance of the metallic structure to be in the frequency region of Raman excitation/scattering. This can be achieved by selecting an appropriate metal (usually this is gold, silver or copper [7]) and tailoring the size and the shape of its surface. In case of nanoparticles, widely used as SERS agents [8], it was found that the peak of the plasmon resonance is shifting to lower frequencies with size [9]; the use of nanorods instead of spheres allows to create structures with dual plasmons resonance frequencies [10, 11]. Colloidal suspensions are also widely used for SERS enhancement, where the size, shape, type or composition of the colloidal structures leads to the tunability of plasmonic resonance [12]. 
In addition to nanostructures, periodic arrays of micron sized voids were found to have surface enhancement properties as well. Producing 3D surfaces with different micro-electromechanical (MEMS) technologies is a very popular approach $[13,14,15]$, where the properties can be tuned by changing the diameter and periodicity of the structures [16], [17]. SERS substrates consisting of gold coated periodic arrays of micron-sized voids are widely used in many fields of analytics [18]. Inverse pyramid shaped voids available commercially as well [19]. Their perforated versions suitable for flow-through applications and micro-object entrapment were fabricated also by us earlier [20,21].

Creation of hotspots by forming gaps between closely spaced SERS surfaces was found to be a highly efficient way to utilize surface enhancement, where the localized near-field intensity can reach very high values. Several methods have been proposed to fabricate such hotspots. One approach uses self-assembled structures for achieving strong near-fields [22, 23]. Close packed nanoislands were also generated by thermal annealing thin layers of gold [24]. There are some studies for hotspot prediction by machine learning $[25,26]$ as well.

Hotspots can be fabricated by combining microscopic SERS surfaces with nanoparticles. In an inverse pyramid containing a nanosphere gaps of continuously increasing size are formed around the contact points of the surfaces, between the curved surface of the nanoparticle and the flat side of the pyramid. In this paper we report on the giant SERS amplification observed in such gold structures. The experimental findings are interpreted by results of modeling the near field distribution for different number of gold nanoparticles placed inside the gold coated inverse pyramid structure.

\section{Methods}

\section{Sample preparation}

Inverse pyramid array SERS substrates were prepared by lithographic technique (see ref. [27] for details). The base size of the pyramids is $2 \times 2$ microns, with 0.15 microns thick gold coating. Gold nanoparticles (GNPs) of $50 \mathrm{~nm}, 100 \mathrm{~nm}, 200 \mathrm{~nm}$ and $250 \mathrm{~nm}$ diameter were purchased form Corpuscular Inc. (www.microspheres-nanospheres.com). A small amount of GNP water suspension was dripped on the array surface. After water evaporation GNPs were settled on the surface, and some of them were entrapped in the voids. In order to keep only the latter, the surface was washed with a few drops of distilled water. Fig. 1 shows the scanning electron microscopic image of a typical area of the SERS substrate where several $200 \mathrm{~nm}$ large GNPs are sitting in the bottom of inverse pyramids.
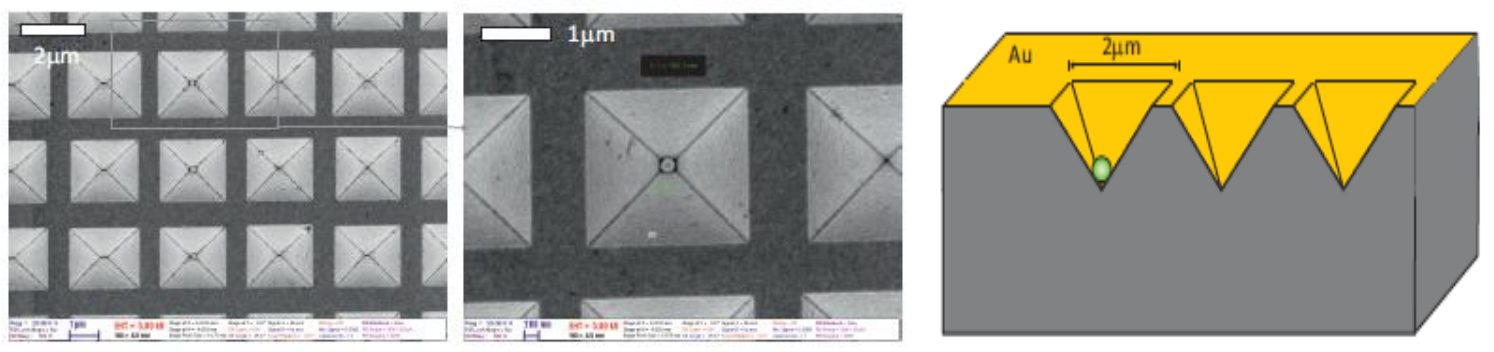

Figure 1. (Left and center) SEM images of the inverse pyramid SERS substrate with $200 \mathrm{~nm}$ GNPs located in some of the voids. (Right) Schematic representation of the SERS substrate with the nanoparticle.

\section{Raman measurements}


Raman spectra were recorded on a Renishaw inVia micro-Raman spectrometer attached to a Leica microscope. A $785 \mathrm{~nm}$ diode laser was used as excitation source and the laser beam was focused into a spot of $1 \mu \mathrm{m}$ diameter on the sample. Inverse pyramids with GNP inside were found by scanning the array surface. Giant SERS amplification was observed when the excitation spot was focused into the center of the inverse pyramid with GNP. These voids were marked and the presence of GNP was verified with scanning electron microscopy (SEM, recorded with a Zeiss LEO 1540XB device).

A $0.1 \mathrm{mM}$ benzophenone solution in ethanol was used to determine the SERS enhancement of the substrates. Raman peak intensities of the compound measured on flat gold substrate were used as reference for the calculations and the SERS enhancement factor was determined from the intensity of the $1598 \mathrm{~cm}^{-1}$ peak.

\section{Determination of the near-field intensity distribution}

The near-field intensity distribution of the inverse pyramid substrates with GNP of different size was studied using the Lumerical FDTD Solutions v.8.15.736 software. Periodic boundary conditions were chosen in the directions parallel to the substrate surface in order to model the array geometry, together with perfectly matched layer boundary conditions in the perpendicular direction. The cell size (including a void and the spacing) was set to $2.9 \times 2.9 \times 4.6 \mu \mathrm{m}$, according to the geometry of the fabricated array structure (Fig. 2). Silicon was selected as substrate material and a $150 \mathrm{~nm}$ gold coating was placed on that. GNPs of different size were placed on the bottom of the inverse pyramid. The software's built in material parameter set from CRC Handbook of Chemistry and Physics [7] was used for the gold layer and the GNP and that from the Handbook of Optical Constants of Solids I [8], for the silicon substrate. The simulation grid was defined by the built-in auto mesh algorithm with an accuracy level of 3 . The substrate was illuminated using a broadband $(400-900 \mathrm{~nm}$ ) plane wave having normal incidence to the array surface and polarization being parallel to the base of the pyramids and the XZ plane. Near-field profiles were recorded in two monitor planes placed along the two symmetry axes of the pyramid (XZ and YZ planes) being perpendicular, and in a third one (XY plane) located above and being parallel to the array surface (see Fig. 2). Another two monitors were placed below and above the substrate to measure the reflection spectrum of the structure.
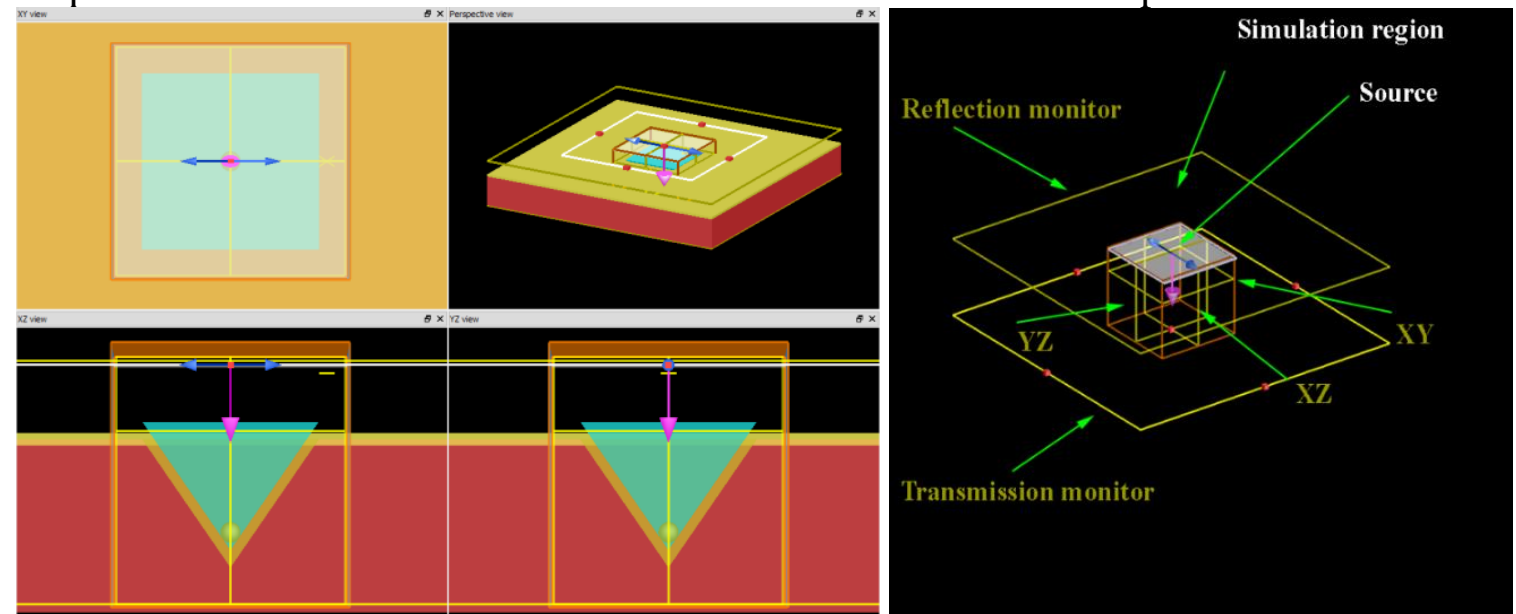

Figure 2. Schematic model of an inverse pyramid cell with entrapped $250 \mathrm{~nm}$ GNP used for modeling. 


\section{Results and discussion}

\section{a. SERS enhancement of inverse pyramids with GNPs}

Fig. 3 below compares three Raman spectra recorded on benzophenone dripped and dried on flat gold surface used as reference, and on the inverse pyramid array surface (without entrapped GNP). Only some of the most intense benzophenone peaks (located at $1000 \mathrm{~cm}^{-1}$ and $1600 \mathrm{~cm}^{-1}$ ) can be detected in the reference spectrum measured on the flat surface, while a strong benzophenone SERS spectrum can be detected on the inverse pyramid array. The observed Raman peaks are in good agreement with the reference spectrum. Comparison of the peak intensities shows that the SERS-amplification of the array is around 2-3 orders of magnitude, relatively to the reference spectrum, and the peak intensities measured on the SERS substrate are at the $10^{5}$ level on average.

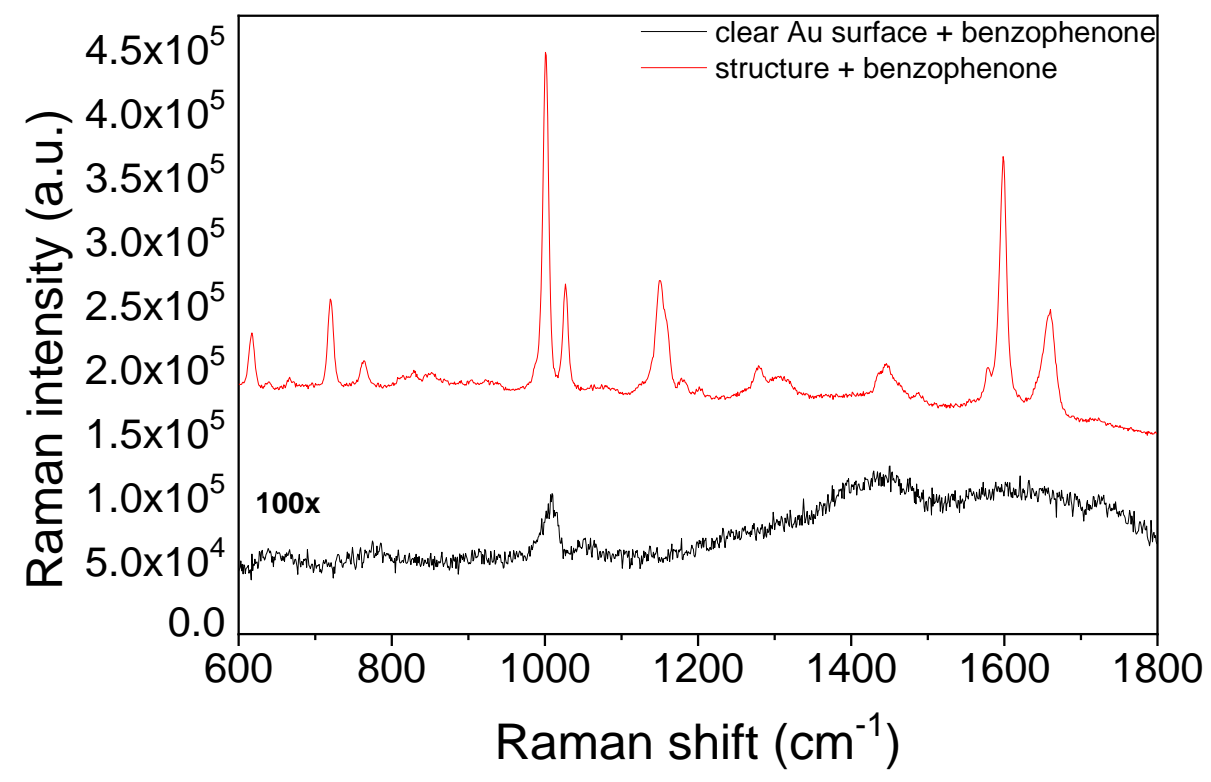

Figure 3. Raman spectra of benzophenone solution measured after dropping and drying on clean flat gold and on inverse pyramid surface.

The same Raman measurements were repeated on inverse pyramids with entrapped GNPs. Fig. 4 compares the rough and baseline corrected surface enhanced Raman spectra recorded with entrapped GNP of different size. It can be seen that the Raman peak intensities are significantly higher compared to the array with empty voids. While it is at the level of $10^{5}$ for the latter (see Fig. 3), a 2 orders of magnitude increase can be observed for the structures with entrapped GNP. In addition, the measured intensity is strongly dependent on the GNP size. While the average level is below $10^{6}$ for $50 \mathrm{~nm}$ GNP, it increases to almost $5 \times 10^{7}$ for the $250 \mathrm{~nm}$ particle. It should be noted that all measurements were performed under the same conditions, with the same analyte and by focusing the laser beam into the center of the inverse pyramid.

On the other hand, the shape of the Raman spectra is different from the benzophenone reference (Fig. 3). New peaks of high intensity are present, and their number, position and relative intensity varies from sample to sample. Additional SERS measurements were performed with freshly prepared benzophenone solution for verification and led to the same result. This variation of Raman peaks resembles the SERS blinking effect [28], however, within a given void the spectrum does not change with time. In spite of the latter we assume that the observed phenomenon is arising from the same effects, as blinking, namely excitation of molecules into 
a metastable and non-emitting state [29, 30]; photoionization affecting charge transfer states [31]; change in orientation of the molecules [32]; photoinduced electron transfer between the adsorbed molecule and the SERS surface [33]; diffusion of molecules at hotspots [34];

The composition of the enhanced signal is also different from that recorded in the empty void. The narrow Raman peaks are sitting on a broad background that can be attributed to photoluminescence (PL). The relative intensity of Raman peaks compared to the PL background decreases with GNP size. While the strongest Raman bands can appear in the spectrum recorded on the $100 \mathrm{~nm}$ sample, that of the $250 \mathrm{~nm}$ sample is dominated by the PL signal. It is known that in addition to SERS, the surface enhancement works for PL process as well [9]. However, while the strongest contribution to SERS comes right from the plasmonic surface [29], the PL is quenched there, and the surface enhancement of photoluminescence has maximum at some distance. Therefore, from the spectra shown on Fig. 4 it can be concluded, that the strongest amplification in samples with larger GNPs is related to PL enhancement and comes from volumes being at some distance from the metallic surface.
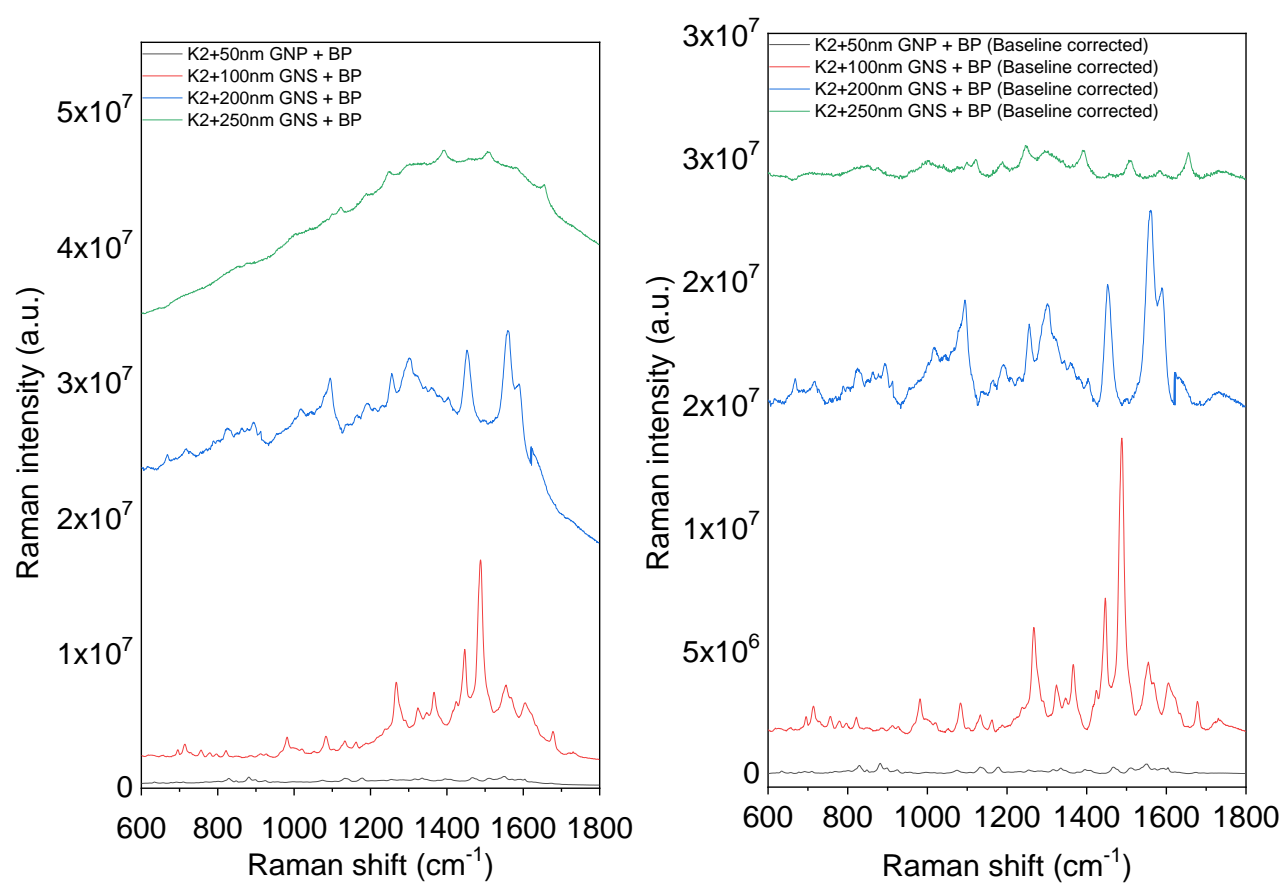

Figure 4. Raw (left) and baseline corrected surface enhanced Raman spectra of benzophenone solution dripped and dried onto inverse pyramid arrays entrapping a GNP of different size.

It was found that the SERS enhancement factor inverse pyramid containing GNP is 2-3 orders of magnitude higher than that of the empty void and the difference is due to the presence of the nanoparticles (which is supported also by the size dependence of the enhancement). Geometrically, this structure is a four-faced pyramid enclosing a sphere. There are four tangential points of pyramid's flat surfaces and the curved surface of the sphere. A gap of cylindrical symmetry is formed around these points, the size of which is increasing with the distance from the contact point. These spaces of variable geometry between the flat and curved gold surfaces are ideal for hotspot formation and giant enhancement of the SERS and PL signals. 
The larger the radius of a sphere, the smaller the curvature of its surface. Assuming that hotspots are forming in gaps below certain size, the structure with $250 \mathrm{~nm}$ GNP will have larger volumes suitable for this, compared to the $50 \mathrm{~nm}$ one. And therefore, the surface enhanced signal will be stronger from the former. Further, since the structure with $250 \mathrm{~nm}$ GNP will have larger hotspot zone volume to surface ratio, the PL signal, coming from the volume, will be stronger than the SERS contribution, arising from the surface (of course, this is a simplified picture of SERS and PL active regions).

As it was discussed earlier, the spectra in Fig. 4 are different from that of benzophenone and from each other, including the number, position and intensity of the Raman peaks. These Raman peaks could arise from: 1) impurities in the analyte and/or on different inverse pyramid surfaces; 2) different impurities on the surface of gold nanospheres of different size; 3) strong field gradients (with magnitude being dependent on the GNP size) in the gap between the pyramid wall and the nanoparticle surface activating new and/or modified Raman transitions of the analyte. The inverse pyramid substrates were fabricated simultaneously, on the same silicon wafer and under the same conditions; the same solution was used for every SERS measurement. Therefore, the impurities (if any) in the solution and on the SERS substrates should also be the same, as well as their Raman peaks in the measured SERS spectra (except the intensity). Suspensions of gold nanoparticles were purchased from the same manufacturer and it can be assumed that they were prepared by using the same technology and they have the same molecules, functional groups, impurities (if any) on their surface. So, the first two reasons described above can be ruled out and the differences in peak positions and intensities in the SERS spectra are due to differences in local field gradients. FDTD simulations were performed in order to determine the magnitude and spatial distribution of the near-field intensity of these structures.

\section{b. Calculated near-field intensity distributions}

Figure 5 compares the calculated near-field intensity in the three monitor planes described above. For comparison, the data obtained for the empty void are provided as well. It can be seen that the local hotspots in the empty pyramid are around 0.4 microns above the bottom of the void. There are two hotspots at this level in the $\mathrm{XZ}$ plane, at the faces of the pyramid, spanning over a 0.4 micron region vertically and having maximum intensity of 8.0. (It should be noted that each map in Fig. 5 has its own intensity scale, even those obtained for the same structure). These hotspots extend to some extent to the YZ plane as well, where they have 5.0 maximum intensity. The XY plane above the void has even smaller intensities, with the maximum magnitude being only 3.0. Here the hotspot zone is above the center of the void and it is symmetric in both $\mathrm{XZ}$ and $\mathrm{YZ}$ planes.

Data obtained for the $50 \mathrm{~nm}$ and $100 \mathrm{~nm}$ samples are very similar to the empty pyramid. The near-field intensity distributions and even the magnitudes are nearly the same. There is some minor difference on the bottom of the void, around the nanoparticle in the $100 \mathrm{~nm}$ sample, where hotspots of small intensity are formed.

Different distribution maps can be seen for larger GNPs. The intensity distribution in the XY plane calculated for the $200 \mathrm{~nm}$ sample is still like the above described ones. In the YZ plane the picture is affected by the nanosphere at the bottom, where it cuts into the hotspot, but the magnitude is still in the level of the empty void. In contrast, significant changes can be seen in the XZ plane, where the most intense hotpots are localized in close vicinity of the GNP and the intensity is an order of magnitude higher than in previous cases (50.0 vs. 8.0). The areas of highest intensity are in the tangential region of the nanosphere and the face of the pyramid. This 
hotspot concentration is more pronounced in the case of the $250 \mathrm{~nm}$ sample, where the maximum intensity is 130. Here the effect of the GNP is more remarkable in XY and YZ planes as well, but the main changes appear in the plane being parallel with the polarization of the incident beam.

Calculations show that the near-field intensity distribution maps can be divided into two groups. In the first, consisting of the $50 \mathrm{~nm}$ and $100 \mathrm{~nm}$ samples, the presence of the nanoparticle has minor effect on the hotspots and their near-field intensity. In the second, formed by the $200 \mathrm{~nm}$ and $250 \mathrm{~nm}$ GNPs, strongest hotspots are forming in close vicinity of the nanoparticle, near its tangential point with the pyramid surface. The analysis of distribution maps (Fig. 5) shows that the main difference between the two groups is the relation of the GNP size to the height of the hotspots in the $\mathrm{XZ}$ plane of the empty inverse pyramid. The latter is 0.4 microns above the bottom of the void and spans 0.4 microns vertically. $50 \mathrm{~nm}$ and $100 \mathrm{~nm}$ particles are well below this hotspot zone (second and third row in Fig. 5). The larger two GNPs are, however, interfere with it and coupling of the electromagnetic field takes place. As a result, new hotspots form near the nanosphere, the maximum intensity of which is much higher than that of the original hotspots of the empty pyramid. Presumably this phenomenon is responsible for the experimentally observed giant SERS enhancement.

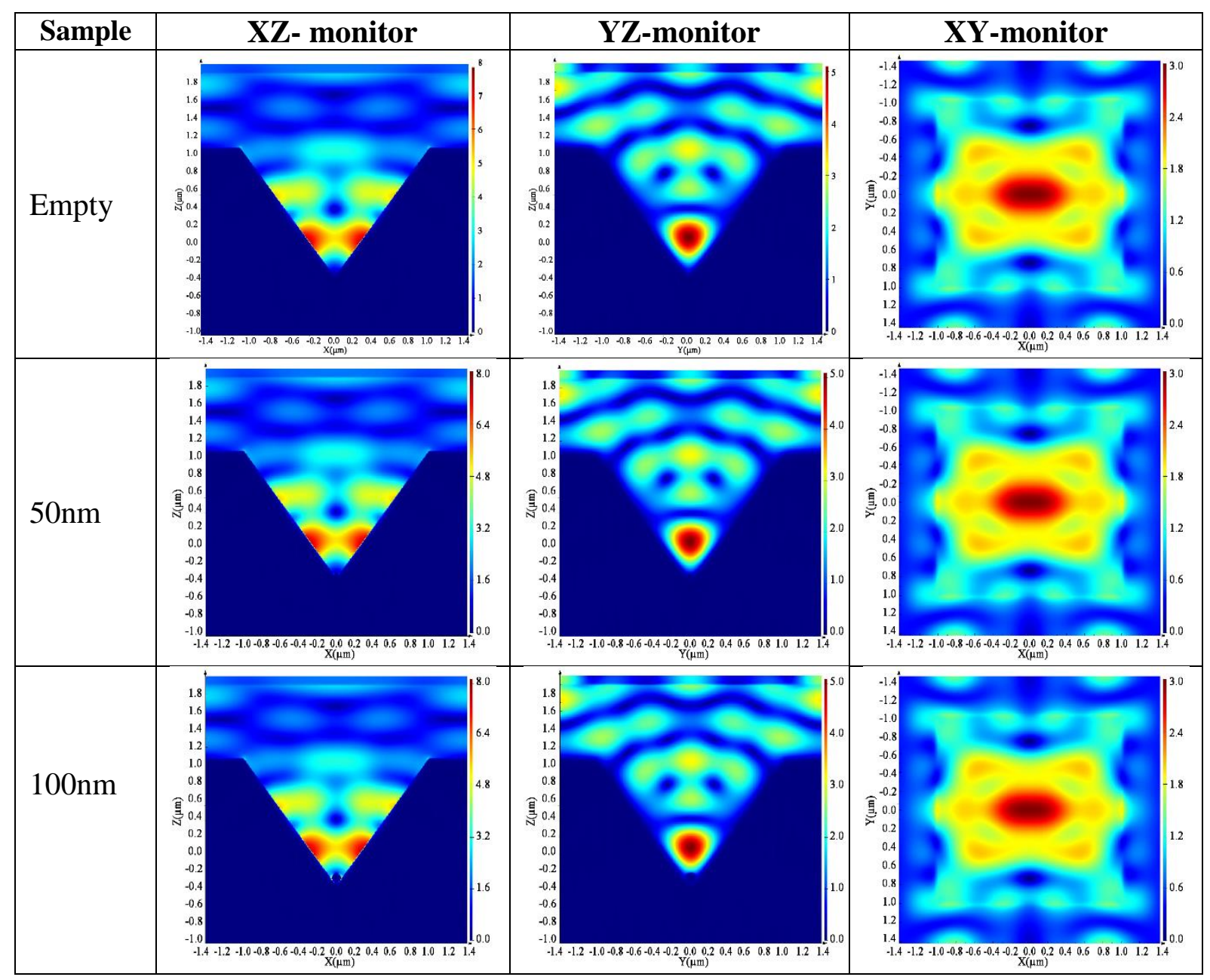




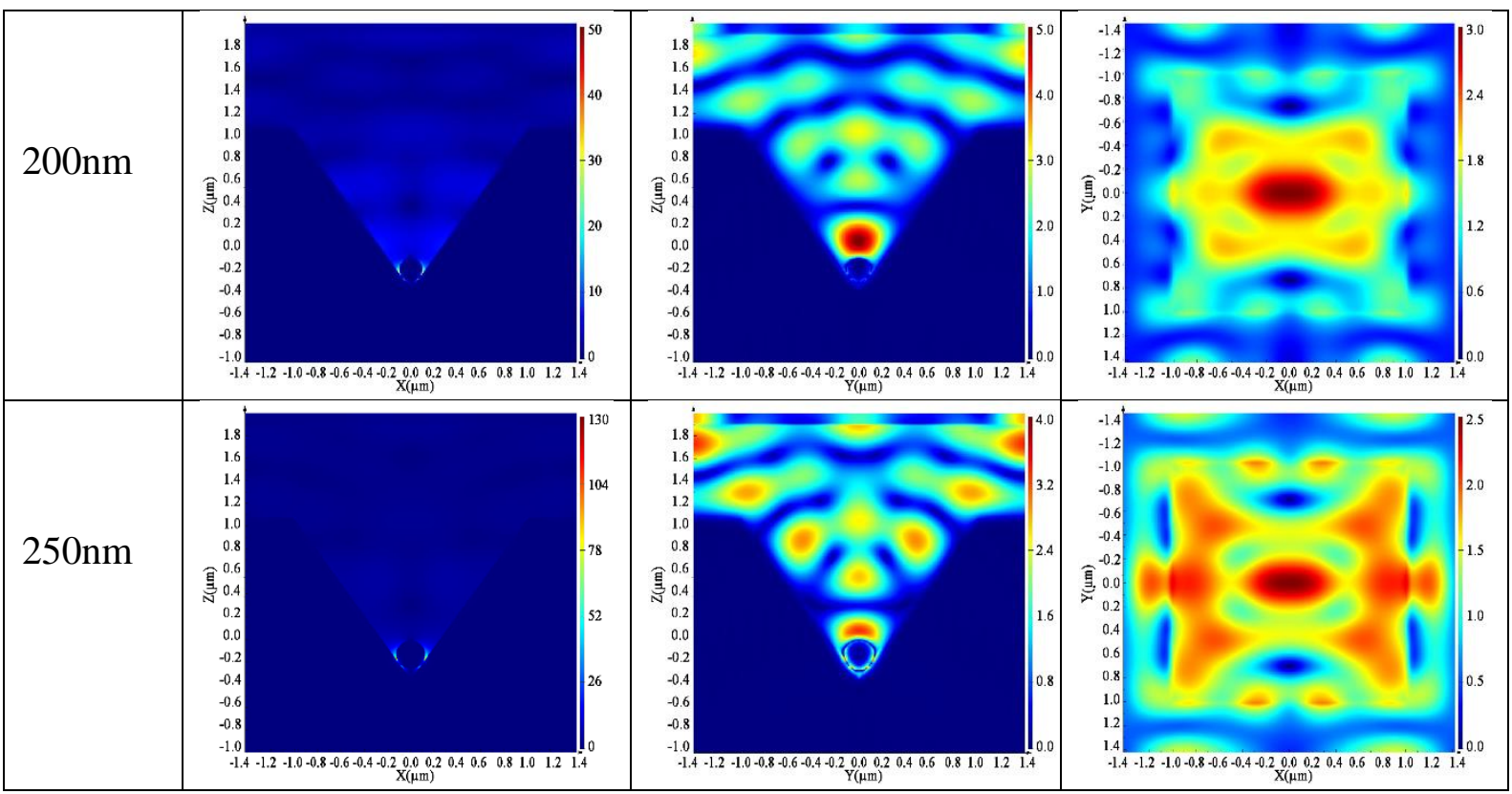

Figure 5. Near-field intensity distributions of the empty inverse pyramid, and that entrapping a 50, 100, 200 and $250 \mathrm{~nm}$ gold nanospheres.

The maximum values of the near-field intensity together with the surface enhancement factors (SEF) determined from the experimentally measured spectra are shown in Figure 6. As it can be expected from the above discussion, the empty, $50 \mathrm{~nm}$ and $100 \mathrm{~nm}$ samples have similar maximum values, while $200 \mathrm{~nm}$ and $250 \mathrm{~nm}$ ones are different. The highest intensity obtained for the $250 \mathrm{~nm}$ sample (130.0) is more than 15 times larger than that of the empty inverse pyramid (around 8.00).

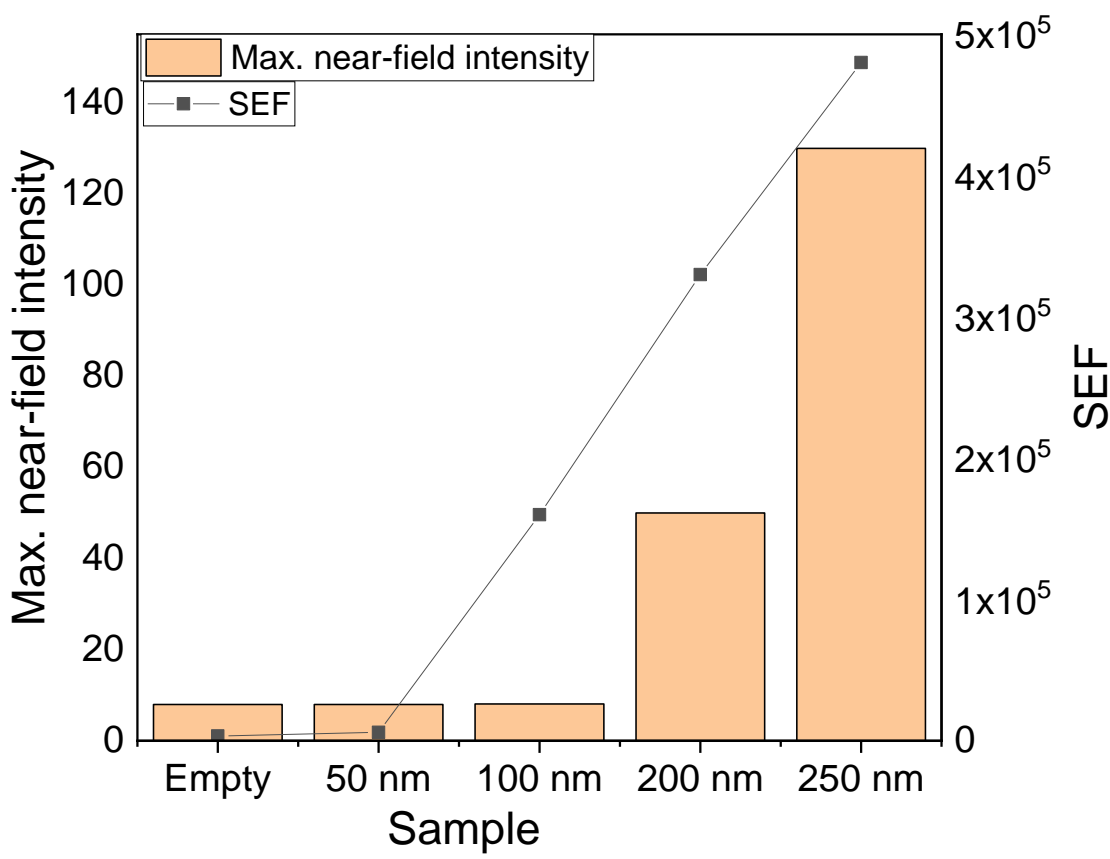

Figure 6. Maxima of the calculated near-field intensity and the surface enhancement factor $(S E F)$ obtained from experimental data for inverse pyramids without and with entrapped gold nanospheres of different size. 
Figure 6 shows also, that the experimentally measured enhancement factor also increases with GNP size, but it behaves differently: the increase is almost linear. This can arise from the discrepancies between the modelled and real parameters of the void and the GNP (like dimensions, shape or the uniformity of the gold coating inside the pyramid) and from the determination of the surface enhancement factor. Raman measurements were performed with $785 \mathrm{~nm}$ excitation, so the SEF was calculated from the spectrum recorded in the 790-900 nm wavelength region, namely around $1400-1500 \mathrm{~cm}^{-1}$ relative wavenumbers (corresponding to 880-890 nm). In contrast, the near-field distribution was determined for a broader spectral range. Figure 7 show the reflection spectrum of the samples obtained from FDTD simulations. While flat gold surface has almost constant reflection in this region, curves in Fig. 7 fluctuate with wavelength. Their local minima correspond to plasmon resonances. While the $50 \mathrm{~nm}$ and $100 \mathrm{~nm}$ samples behave very similarly and have a remarkable minimum around $820 \mathrm{~nm}$, stronger plasmon coupling can be seen in the case of the $200 \mathrm{~nm}$ and especially $250 \mathrm{~nm}$ sample. Both have a minimum around $820 \mathrm{~nm}$, but the $250 \mathrm{~nm}$ has another one at $860 \mathrm{~nm}$ as well. In addition to discrepancies between the modelled and real structures the behavior of the measured SEF values can be affected by these differences as well.

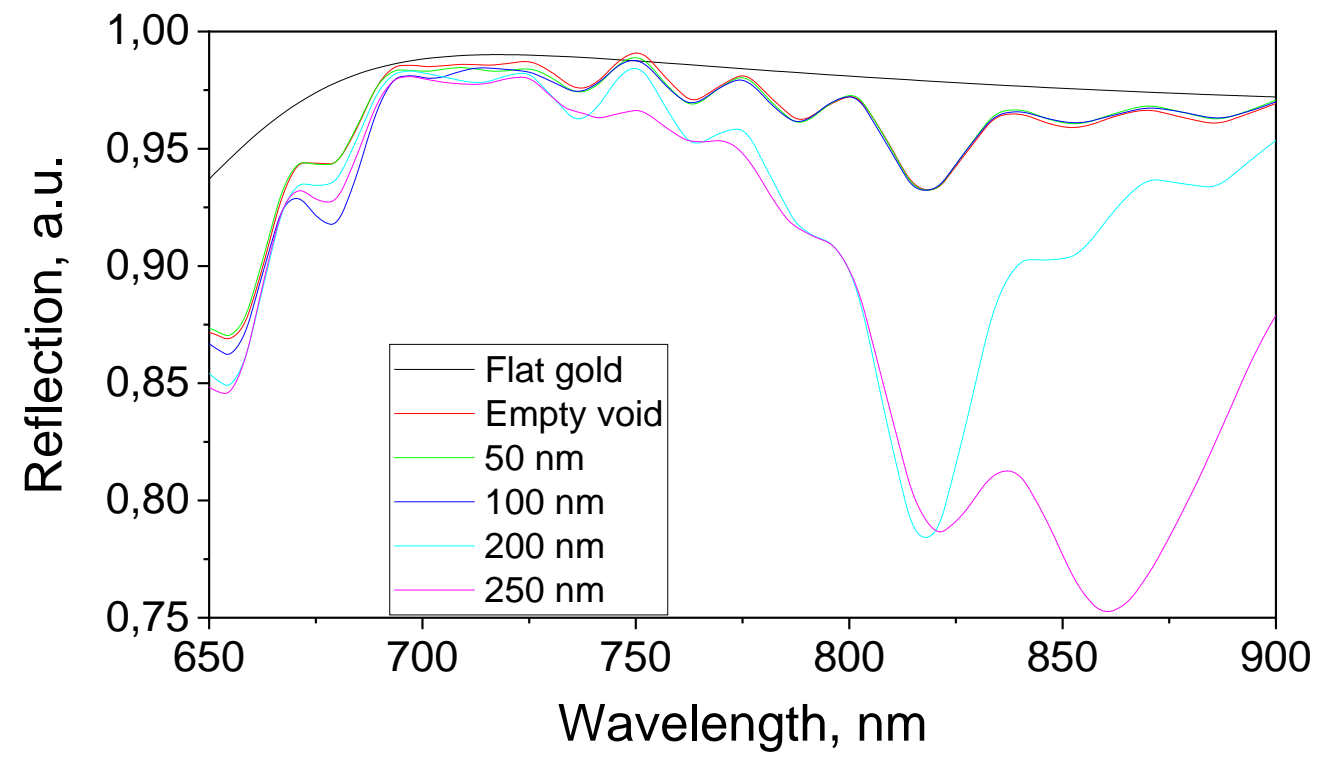

Figure 7. Optical reflection spectra of inverse pyramids containing gold nanospheres of different size, obtained by FDTD modeling.

\section{Conclusions}

A remarkable increase of the surface enhancement has been observed in gold coated micron sized inverse pyramid SERS substrate after entrapping a gold nanoparticle in it. The amplification of both surface enhanced Raman and photoluminescence signals was found to be dependent on the size of the gold nanoparticle and the enhancement with a $250 \mathrm{~nm}$ nanosphere was 50 times larger than that of the empty pyramid. The origin of the phenomenon was investigated by finite differential time domain simulations and they showed that coupling of electric field occurs when the nanoparticle protrudes into the hotspot zone of the inverse pyramid. The plasmon-related near-field enhancement is concentrated into the close gaps around the tangential points of the curved sphere and the flat pyramid surface.

\section{Acknowledgements}


This work was supported by the VEKOP-2.3.2-16-2016-00011 grant, which is co-financed by the European Union and European Social Fund.

\section{References}

[1] " C. Kumar, Raman Spectroscopy for Nanomaterials Characterization, Springer, Heidelberg, 2012, pp. 215e254. Verlag Berlin".

[2] "A.G. Ryder, Surface enhanced Raman scattering for narcotic detection and applications to chemical biology. Curr. Opin. Chem. Biol. 9 (2005) 489-493.".

[3] "P. Etchegoin, R.C. Maher, L.F. Cohen, H. Hartigan et al., New limits in ultrasensitive trace detection by surface enhanced Raman scattering (SERS). Chem. Phys. Lett 375 (2003) 84-90.".

[4] "Kneipp, K.; Wang, Y.; Kneipp, H.; Perelman, L. T.; Itzkan, I.;Dasari, R. R.; Feld, M. S. Single Molecule Detection Using Surface-Enhanced Raman Scattering (SERS). Phys. Rev. Lett. 1997, 78, 1667-1670.".

[5] "Martín-Yerga, Daniel, et al. "Towards single-molecule in situ electrochemical SERS detection with disposable substrates." Chemical Communications 54.45 (2018): 5748-5751.".

[6] "Le Ru, Eric, and Pablo Etchegoin. Principles of Surface-Enhanced Raman Spectroscopy: and related plasmonic effects. Elsevier, 2008.".

[7] "Sharma, Bhavya, et al. "SERS: Materials, applications, and the future." Materials today 15.1-2 (2012): 16-25.".

[8] "He, Rui Xiu, et al. "Effect of the size of silver nanoparticles on SERS signal enhancement." Journal of Nanoparticle Research 19.8 (2017): 267.".

[9] "Etchegoin, Pablo G., and Eric C. Le Ru. "Basic electromagnetic theory of SERS." Surface Enhanced Raman Spectroscopy (2011): 1-37.".

[10] "Mano, Ryoya, et al. "Tuning the Resonant Frequency of a Surface Plasmon by Double-Metallic $\mathrm{Ag} / \mathrm{Au}$ Nanoparticles for High-Efficiency Green Light-Emitting Diodes." Applied Sciences 9.2 (2019): 305.".

[11] "Wang, Junqiao, et al. "Theoretical investigation of a multi-resonance plasmonic substrate for enhanced coherent anti-Stokes Raman scattering." Optics express 25.1 (2017): 497-507.".

[12] "Njoki, Peter N., et al. "Size correlation of optical and spectroscopic properties for gold nanoparticles." The Journal of Physical Chemistry C 111.40 (2007): 14664-14669.".

[13] "Suresh, Vignesh, et al. "Fabrication of Large-Area Flexible SERS Substrates by Nanoimprint Lithography." ACS Applied Nano Materials 1.2 (2018): 886-893.".

[14] "Tan, Joel Ming Rui, et al. "A large-scale superhydrophobic surface-enhanced Raman scattering (SERS) platform fabricated via capillary force lithography and assembly of Ag nanocubes for ultratrace molecular sensing." Physical Chemistry Chemical Physics 16.". 
[15] "Hwang, June, and Minyang Yang. "Sensitive and Reproducible Gold SERS Sensor Based on Interference Lithography and Electrophoretic Deposition." Sensors 18.11 (2018): 4076.".

[16] "István Rigó, Miklós Veres, László Himics, Sára Tóth, Aladár Czitrovszky, Attila Nagy, Péter Fürjes; Comparative Analysis of SERS Substrates of Different Morphology; PROCEDIA ENGINEERING 168 pp. 371-374., (2016).".

[17] "Yu, Qiuming, et al. "Inverted size-dependence of surface-enhanced Raman scattering on gold nanohole and nanodisk arrays." Nano letters 8.7 (2008): 1923-1928.".

[18] "Oh, Young-Jae, et al. "Engineering hot spots on plasmonic nanopillar arrays for SERS: A review." Biochip Journal 10.4 (2016): 297-309.".

[19] "Renishaw Diagnostics Limited (RDL) Klarite ${ }^{\circledR}, " \quad$ [Online]. Available: https://www.renishaw.com/en/eu-project-delivers-new-possibilities-for-sers-sensors--24460.

[20] "István Rigó, Miklós Veres, Péter Fürjes; SERS Active Periodic 3D Structure for Trapping and High Sensitive Molecular Analysis of Particles or Cells; PROCEEDINGS 1 : 4, (2017).".

[21] "István Rigó, Miklós Veres, Tamás Váczi, Eszter Holczer, Orsolya Hakkel, András Deák, Péter Fürjes; Preparation and Characterization of Perforated SERS Active Array for Particle Trapping and Sensitive Molecular Analysis; Biosensors 9 (3), 93 (2019)".

[22] "Zhang, Qi, et al. "Hierarchical 3D SERS Substrates Fabricated by Integrating Photolithographic Microstructures and Self-Assembly of Silver Nanoparticles." Small 10.13 (2014): 2703-2711.".

[23] "Fan, Meikun, and Alexandre G. Brolo. "Silver nanoparticles self assembly as SERS substrates with near single molecule detection limit." Physical Chemistry Chemical Physics 11.34 (2009): 7381-7389.".

[24] "Bonyar, Attila, et al. "Investigation of the performance of thermally generated gold nanoislands for LSPR and SERS applications." Sensors and Actuators B: Chemical 255 (2018): 433-439.".

[25] "Wuu, Jen-Yi, Fedor G. Pikus, and Malgorzata Marek-Sadowska. "Efficient approach to early detection of lithographic hotspots using machine learning systems and pattern matching." Design for Manufacturability through Design-Process Integration V. Vol. 7974.".

[26] "Matsunawa, Tetsuaki, Shigeki Nojima, and Toshiya Kotani. "Automatic layout feature extraction for lithography hotspot detection based on deep neural network." Design-Process-Technology Cooptimization for Manufacturability X. Vol. 9781. International Soci".

[27] "Rigó, I., et al. "Preparation and Characterization of SERS Substrates of Different Morphology." Advanced Nanotechnologies for Detection and Defence against CBRN Agents. Springer, Dordrecht, 2018. 63-68.".

[28] "Kitahama, Yasutaka, Yumi Nishiyama, and Yukihiro Ozaki. "Blinking Surface-Enhanced Raman Scattering and Fluorescence From a Single Silver Nanoaggregate Simultaneously Analyzed by Bi-Color Intensity Ratios and a Truncated Power Law." The Journal of Physica".

[29] "Dornhaus, R., Benner, R. E., Chang, R. K., \& Chabay, I. (1980). Surface plasmon contribution to SERS. Surface Science, 101(1-3), 367-373.". 